\title{
Reconfigurable Dielectric Resonator Filters
}

\author{
Mohammed AlQaisei, Abdelfattah Sheta, and Majeed Alkanhal
}

\begin{abstract}
This paper presents a new reconfigurable dielectric resonator (DR) filter. The developed filter structure consists of two coupled reconfigurable cylindrical dielectric resonators. The reconfigurability is achieved using RF switches. RF switches can be implemented using either PIN diodes switches or microelectromechanical switches (MEMS). A switchable $36 \mathrm{MHz}$ bandwidth ( $1.5 \%$ relative bandwidth) filter is designed for switching between center frequencies of $2.55 \mathrm{GHz}$ and $2.65 \mathrm{GHz}$. Simulation is performed using Computer System Technology (CST) software. The filters characteristics are presented and discussed.
\end{abstract}

Keywords—Reconfigurable, Dielectric, Resonator, Filter.

\section{INTRODUCTION}

$\mathrm{F}$ ILTERS are the most common devices that determines the operating frequency of any communication system and they become crucial elements especially in multiband-based communication systems. Microwave filters are essential components in radar, satellite, and mobile communication systems. Reconfigurable filters have recently attract much attention to accommodate a wide band of frequencies in multi standards platform. This enable systems to be flexibly switching among multi standards with the ability to fulfill their requirements, on top of them is the operating frequency. Resonators are important and vital components utilized in almost all microwave systems and they are used in filtering, frequency selection, and tuned amplifiers. Typically narrow bandpass devices having stringent specifications for passband insertion loss, stopband rejection, power handling, and physical size.

A dielectric resonator (DR) consists of a cylindrical, cubic or any other shaped piece of high dielectric constant material called a puck [1]. At the resonant frequency, most of the electromagnetic energy is stored within the dielectric and the enclosure of DR structure prevent radiation.

Reconfigurable filters are basically used to avoid the use of bank of filters, thus providing more practical and flexible solutions to almost all the communication radio circuitry. The reconfigurable dielectric resonator filters (DRFs) are widely applied in the modern wireless communication systems due to a wide variety of filters characteristics, such as high $Q$ factor at suitable overall size [2]-[5].

Tunable filters can replace the necessity of switching between several filters to have more than one filter response via introducing tuning elements embedded into the filter topology. Generally speaking, microwave tunable filters can

Authors are with the King Saud University, Riyadh, Saudi Arabia. be divided into two groups; filters with discrete tuning and filters with continuous tuning.

Filter topologies presenting a discrete tuning generally use PIN diodes or Microelectromechanical systems (MEMS) switches. On the other hand, filter topologies that use varactor diodes, MEMS capacitors, ferroelectric materials, or ferromagnetic materials are commonly used to obtain a continuous tuning device. Each tuning approach has a different impact on the performance of tunable filters, including insertion loss, tuning speed, and tuning range. Dielectric resonators have a high dielectric constant and a low dissipation factor, which produces a high quality factor and inturn gives a low insertion loss measurement over the filters passband. Dielectric resonator filters are preferable for wireless base stations due to their superior characteristics of a high quality Q factor and miniaturization [5]. A dielectric materials such as ceramic, are the basic element of configured dielectric resonators. These materials are inherently designed to act as resonators for radio waves that can be used in wireless communication systems [6], [7].

Reconfigurable dielectric resonators filters are very difficult to be implemented. As far as we know, the only suggested tunable dielectric resonator filters configuration is that based on MEMS technique by electronically adjusting the size of the cavity [4]-[5]-[8]. This technique is very complex and expensive. In this paper a new technique is developed that allows the use of a simple low cost PIN diode switches to adjust electronically the filter frequency. The design and implementation of narrow band tunable dielectric resonator filter is presented and discussed. Simulation is performed using Computer System Technology (CST) software.

\section{RECONFIGURABLE RESONATOR}

The proposed reconfigurable resonator is based on a cylindrical dielectric resonator made of ceramic material with dielectric constant $\varepsilon_{\mathrm{r}}=20$. The resonator diameter and length are $16.2 \mathrm{~mm}$, and $17 \mathrm{~mm}$, respectively, and is placed on a Teflon disk of the same diameter with $\varepsilon_{\mathrm{r}}=2.2$ and thickness $1.6 \mathrm{~mm}$. This Teflon disk is mainly used to align the DR during the process of assembly. The resonator is centered inside a metallic cavity. The metallic cavity is $28 \mathrm{~mm}$ in length, $28 \mathrm{~mm}$ in width, and $20.38 \mathrm{~mm}$ in height and it is made of Aluminum. These dimensions are selected to adjust the resonance frequency at approximately $2.5 \mathrm{GHz}$. These dimensions can be modified in such a way to get any specific resonance frequency. The excitation is attained by a coaxial probe, acting as an input port and it is extended very closely to the DR. On the opposite side of the input port is placed 
another coaxial probe acting as an output port and the DR is located at the center of the metallic cavity as shown in Fig. 1.

The top side of the metallic cavity is replaced by a single sided Teflon rectangular dielectric substrate with dielectric constant $\varepsilon_{\mathrm{r}}=4.2$ and thickness $1.6 \mathrm{~mm}$. Two slots are etched on the top side as shown in Fig. 1. The first is a circular slot of $2 \mathrm{~mm}$ radius. The second slot is a circular ring of inner and outer radii $4 \mathrm{~mm}$ and $6 \mathrm{~mm}$, respectively. However, as mentioned previously the other side of this substrate, faced the dielectric resonator, is free from any metallic parts. A pair of control reconfigurable element (PIN diodes) were then located over the slot as shown in Fig. 2. The PIN diodes electronic switches are implemented in order to connect or disconnect two points together. These two PIN diodes are used to control the resonant frequency.

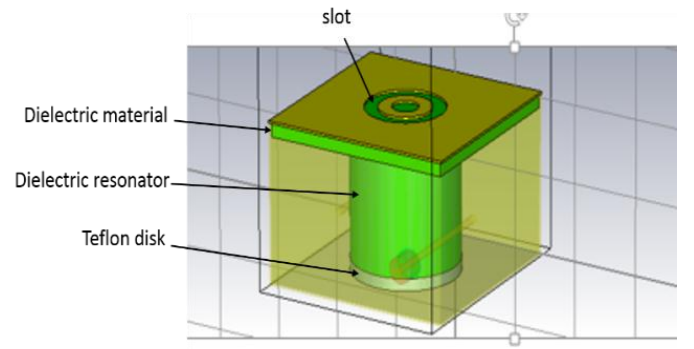

Fig. 1 A 3D view of the components of the designed circular-slot DR

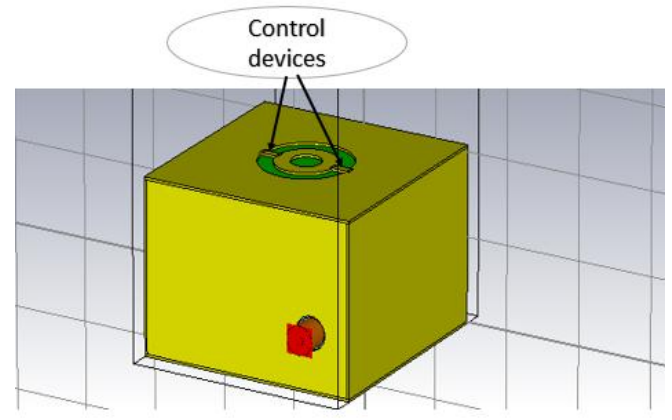

Fig. 2 A 3D view of the designed circular-slot DR with two PIN diodes control devices

The relationship that relates the $\mathrm{Q}$ factor to the fractional bandwidth $(\omega)$ is given by:

$Q=\frac{4.343 n}{I . L \times \omega}$

where $\mathrm{n}$ is the number of resonators and I.L is the insertion loss. The relative bandwidth is given by:

$$
\omega=\frac{3 d \text { Bbandwidth }}{f_{0}}
$$

where $f_{0}$ is the resonant frequency of the filter.

In order to predict the interaction of electromagnetic fields in microwave filter designs, it is vital to use electromagnetic field simulation software. In our design, the CST software is used in order to simulate a single circular-slot resonator.
The mean of reconfigurability is achieved in our design via implementing a pair of PIN diodes switches. These switches are mainly utilized to generate reconfigurable discrete states on the response of the filter, and they have an advantage of providing low cost implementations. When both PIN diodes are switched on, the center frequency of the resonator is found to be $2.53 \mathrm{GHz}$, whereas if both switches are switched off, the center frequency is shifted to $2.64 \mathrm{GHz}$. Fig. 3 and 4. reveal the $S$ parameters $\left(S_{11}\right.$ and $\left.S_{21}\right)$ results for these two cases, respectively.

It is worthy to mention that when changing the length (L) and diameter (D) of the DR, the resonant frequency of the final DR design will also change. For example, if both $\mathrm{L}$ and $\mathrm{D}$ increase, the resonant frequency decreases and vice versa.

Microwave resonators in this work a single mode resonators. A single-mode resonator supports only a single field distribution at the resonator's desired resonance frequency. The transverse filed components in this dielectric resonator is $H E_{11}$ mode.

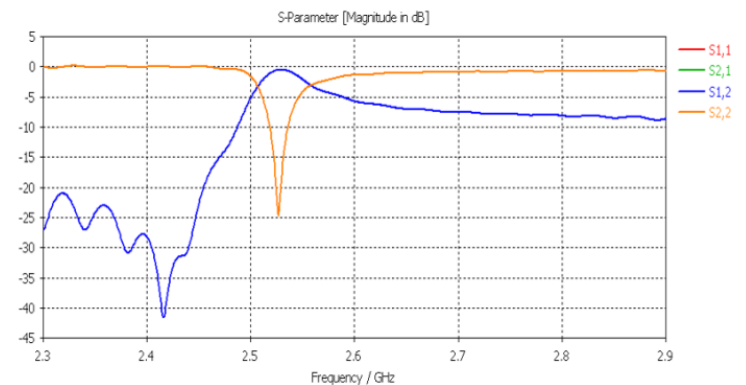

Fig. 3 Simulated results of the circular-slot DR when the PIN switches are on

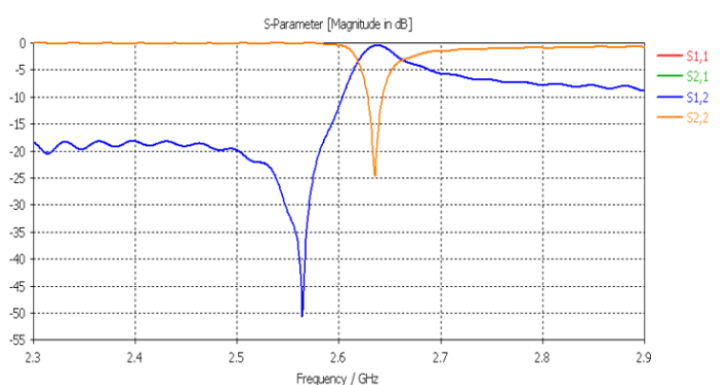

Fig. 4 Simulated results of the circular-slot DR when the PIN switches are off

\section{RECONFIGURABLE FILTER}

It is commonly known that, the simplest topology of two poles bandpass filter consists of typical two coupled resonators. The coupling between the resonators control the filter bandwidth [1]-[9]. Fig. 5 depicts in a 3D view our proposed reconfigurable circular-slot DRF, in which we used four PIN diodes (each DR has a pair of diodes) acting as control devices in order to achieve reconfigurability. The resonators described in the previous section is used. The coupling between the resonators is achieved by a horizontal rectangular hole made in an Aluminium plate of $28 \mathrm{~mm}$ length, $7.5 \mathrm{~mm}$ width, and $20.38 \mathrm{~mm}$ height as shown in Fig. 6. The rectangular hole dimensions are $24 \times 2 \mathrm{~mm}^{2}$. This 
rectangular hole is placed $14 \mathrm{~mm}$ from the bottom. As shown in Fig. 6, the input probe is placed beside the DR with a 6.75 $\mathrm{mm}$ apart from the bottom of the cavity, and an extension of $18 \mathrm{~mm}$ into the cavity. The output probe, on the other hand is placed at exactly the same location but at the opposite side of the other DR.

The designed DRF was developed to operate in two distinctive cases. These two cases are classified based on the state of the quadruple PIN diodes i.e., the first case represents the case when all the four PIN switches are switched on whereas the second case represents the case when all the four PIN switches are switched off. The respective S parameters responses were obtained for each of these two cases.

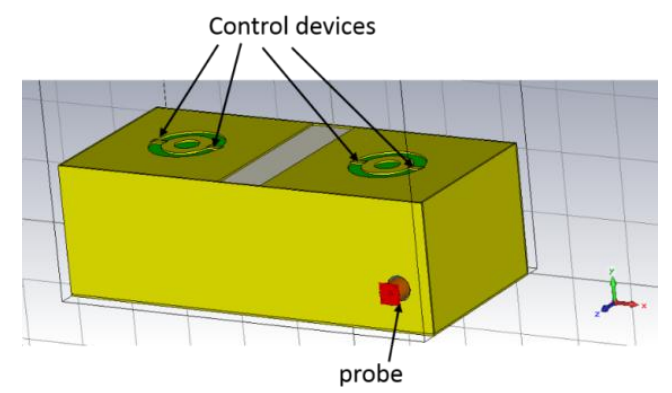

Fig. 5 A CST simulation model for the 2-pole circular-slot DRF with PIN control devices

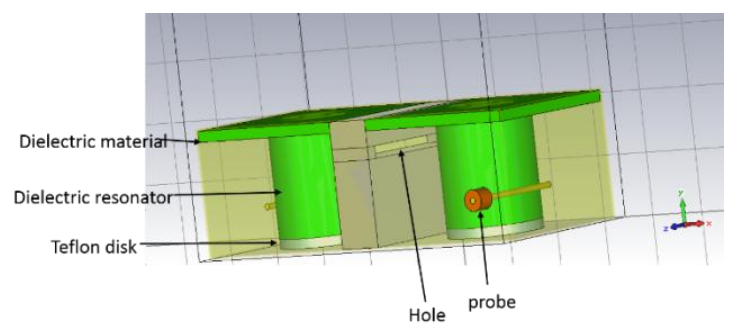

Fig. 6 A CST simulation model for the 2-pole circular-slot DRF

Fig. 7 shows the simulation results of the $S$ parameter response $\left(S_{11}\right.$ and $\left.S_{21}\right)$ for the all-switches-on case. It is clear from this figure that the simulated center frequency is 2.535 $\mathrm{GHz}$ with a $3 \mathrm{~dB}$ bandwidth of $36 \mathrm{MHz}$ (1.4\% relative bandwidth). The mid-band filter insertion loss is $0.4 \mathrm{~dB}$. The respective simulation results of the $\mathrm{S}$ parameter responses of the second case (all-switches-off case) are shown in Fig. 8. It is obvious from this figure that simulated resonant frequency is $2.625 \mathrm{GHz}$ with a $3 \mathrm{~dB}$ bandwidth of $39 \mathrm{MHz}(1.5 \%$ relative bandwidth). An insertion loss of $0.9 \mathrm{~dB}$ is observed.

Experimental validation is under process and will be available in the conference presentation.

\section{CONCLUSION}

In this paper, a new structure for Reconfigurable DR filters is presented. The Reconfigurable mechanism is based on a PCB circuit with a PIN diode placed the top of DR cavity resonator. The reconfigurable DRF was developed based on ring slot configuration. A filter relative bandwidth of about $1.5 \%$ is achieved and can be switched between two center frequencies of $2.55 \mathrm{GHz}$ and $2.65 \mathrm{GHz}$.

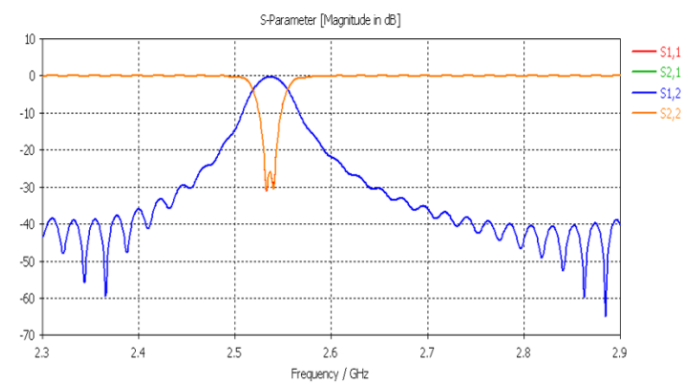

Fig. 7 Simulated results of the circular-slot DRF when the PIN switches are switched on

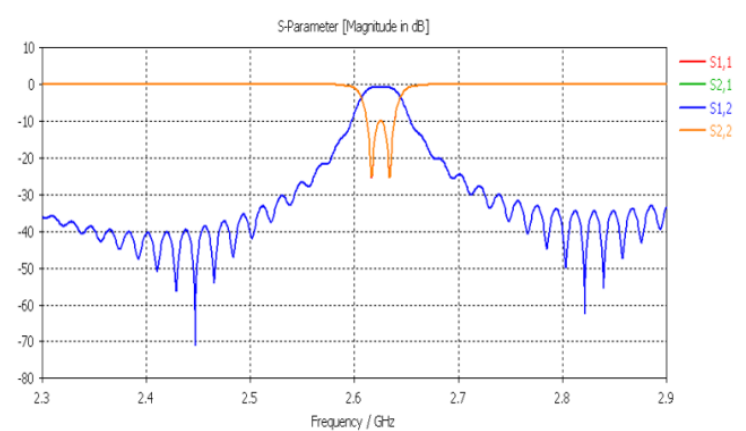

Fig. 8. Simulated results of the circular-slot DRF when the PIN switches are switched off

\section{ACKNOWLEDGMENT}

The authors would like to acknowledge the Deanship of Scientific Research, Research Centre at the College of Engineering, King Saud University for supporting this research.

\section{REFERENCES}

[1] I. Hunter, Theory and design of microwave filters: Iet, 2001.

[2] B. S. Virdee, A. S. Virdee, and L. Trinogga, "Novel invasive electronic tuning of dielectric resonators," in Microwave Symposium Digest, 2003 IEEE MTT-S International, 2003, pp. 51-54. http://dx.doi.org/10.1109/mwsym.2003.1210881

[3] R. R. Mansour, "Filter technologies for wireless base stations," Microwave Magazine, IEEE, vol. 5, pp. 68-74, 2004. http://dx.doi.org/10.1109/MMW.2004.1284945

[4] R. R. Mansour, "High-Q tunable dielectric resonator filters," Microwave Magazine, IEEE, vol. 10, pp. 84-98, 2009. http://dx.doi.org/10.1109/MMM.2009.933591

[5] R. Zhang and R. R. Mansour, "Low-cost dielectric-resonator filters with improved spurious performance," Microwave Theory and Techniques, IEEE Transactions on, vol. 55, pp. 2168-2175, 2007. http://dx.doi.org/10.1109/TMTT.2007.906540

[6] S. B. Cohn, "Microwave bandpass filters containing high-Q dielectric resonators," Microwave Theory and Techniques, IEEE Transactions on, vol. 16, pp. 218-227, 1968. http://dx.doi.org/10.1109/TMTT.1968.1126654

[7] K. Wakino, T. Nishikawa, and Y. Ishikawa, "Miniaturization technologies of dielectric resonator filters for mobile communications," Microwave Theory and Techniques, IEEE Transactions on, vol. 42, pp. 1295-1300, 1994.

http://dx.doi.org/10.1109/22.299721

[8] W. D. Yan and R. R. Mansour, "Tunable dielectric resonator bandpass filter with embedded MEMS tuning elements," Microwave Theory and Techniques, IEEE Transactions on, vol. 55, pp. 154-160, 2007. http://dx.doi.org/10.1109/TMTT.2006.888582

[9] T. V. Pidhurska and A. Trubin, "Dual-bandpass filter built on rectangular dielectric resonators," Radioelectronics and Communications Systems, vol. 58, pp. 174-180, 2015 http://dx.doi.org/10.3103/S0735272715040056. 